\title{
OPEN Endoscopy applications for the second law analysis in hydromagnetic peristaltic nanomaterial rheology
}

\author{
Muhammad Awais ${ }^{1}$, Muhammad Shoaib ${ }^{1}$, Muhammad Asif Zahoor Raja ${ }^{2 \bowtie}$, Saba Arif ${ }^{1}$, \\ Muhammad Yousaf Malik ${ }^{3}$, Kottakkaran Sooppy Nisar ${ }^{4 凶} \&$ Khadiga Ahmed Ismail ${ }^{5}$
}

In current study, analysis is presented for peristaltic motion of applied magnetic field and entropy generation within couple stress $\left(\mathrm{Cu} / \mathrm{H}_{2} \mathrm{O}\right)$ nanofluid through an endoscope. An endoscope contains two coaxial cylindrical tubes in which the internal tube is nonflexible while the external tube has sinusoidal wave passing through the boundary. Influences of mixed convection along with applied magnetic field are encountered as well. Formulated governing model is fabricated introducing long wavelength and creeping Stokesian flow approximation which are then analyzed numerically by utilizing Adams Bashforth method. For a physical insight, results are demonstrated to examine the behaviors of flow profiles and entropy generation number for emerging flow parameters with the help of graphs, barcharts and tables.

Researchers have gained much attention in non-Newtonian fluid behaviours due to its novel applications in physiology, industry, and technological processes. Non-Newtonian fluids possess a nonlinear relationship among the rate of strain and shear stresses. Among the theories of non-Newtonian fluids, the couple stress fluid theory is an important one which is further a subclass of polar fluid theories introduced by Stokes. Constitutive relation that describes the behaviour of couple stress fluids encounter couple stresses along with classical Cauchy stress. Moreover, it is an oversimplification of the conventional theory of Newtonian fluids, which validates polar effects. Such fluids include biological fluids, cosmetics, slurries and dairy wastes etc. Characteristically, Devakar and Iyengar ${ }^{1}$ has been investigated flow dynamics of couple stress fluid configured inside two parallel plates. Geometries of the cylindrical pipes with slip wall conditions and analysis of couple stress fluid transport between the parallel surfaces have been obtained by Devakar et al. ${ }^{2,3}$. Srivastava ${ }^{4}$ analyzed consequences of axially symmetric mild stenosis for blood transport presuming blood as couple stress fluid. In order to inspect the performance of rheological complex fluids, investigations pertaining couple stress fluid are incredibly constructive ${ }^{5-10}$.

Furthermore, since several realistic fluids serve as couple stress fluids and their remarkable applications in heat transfer fields, thermal characteristics can be amplified by suspending particles with nanometer-size called nanoparticles pioneered by $\mathrm{Choi}^{11}$. For instance, Khan et al. ${ }^{12}$ have been investigated couple stress nanofluid flow through an oscillatory stretching sheet presuming the impacts of mixed convection with heat generation/ absorption. Some remarkable applications regarding couple stress nanofluids are ${ }^{13-16}$.

Peristaltic motion has extensive applications in engineering processes, physiology and industry. In many biological systems, peristalsis has become one of the major apparatus for fluid transport, initially investigated by Engelman ${ }^{17}$. Recently, Hayat et al. ${ }^{18}$ have been investigated the impacts of convective conditions and nanoparticles on the peristaltic transport simultaneously. Moreover, the endoscope has many clinical applications. For medical recognition, the endoscope/annulus has important effects on the peristaltic flow. In cancer therapy, for desirable tissues removal, heat transfer is very extensively applicable. For instance, heat transfer in peristaltic flow through a vertical porous annulus has been presented by Vajravela et al. ${ }^{19}$. The closed-form solution of a nanofluid for

\footnotetext{
${ }^{1}$ Department of Mathematics, COMSATS University Islamabad, Attock Campus, Attock 43600, Pakistan. ${ }^{2}$ Future Technology Research Center, National Yunlin University of Science and Technology, 123 University Road, Section 3, Douliou, Yunlin 64002, Taiwan, ROC. ${ }^{3}$ Department of Mathematics, College of Sciences, King Khalid University, Abha 61413, Saudi Arabia. ${ }^{4}$ Department of Mathematics, College of Arts and Sciences, Prince Sattam Bin Abdulaziz University, Wadi Aldawaser 11991, Saudi Arabia. ${ }^{5}$ Department of Clinical Laboratory Sciences, College of Applied Medical Sciences, Taif University, P.O. Box 11099, Taif 21944, Saudi Arabia. ${ }^{\circ}$ email: rajamaz@ yuntech.edu.tw; n.sooppy@psau.edu.sa
} 
the peristalsis in an annular section has been presented by Shahzadi and Nadeem ${ }^{20}$. Entropy generation analysis in the peristalsis of nanofluids due to complex flow structures has motivated the researchers. Entropy production in peristaltically occurred nanofluid flow has been analyzed by Hayat et al. ${ }^{21}$. The generation of entropy for couple stress fluid has been studied by Jangli et $\mathrm{al}^{22}$. Further studies for fluid flows with entropy generation analysis can be seen in references ${ }^{23-25}$.

Magnetohydrodynamic explains the magnetic aspects of electrically conducting fluid and have numerous important usages in controlling the velocity of fluids by implementing magnetic field effects. Recently, Awan et al. ${ }^{26}$ inspected an unsteady hydro-magnetic nanofluid flow and heat transfer numerically through the channel. Simulation of computational fluid dynamics for suspension of nanoparticles in MHD liquid has been analyzed by Nawaz et al. ${ }^{27}$. Some ongoing research can be seen through the references ${ }^{28-30}$.

By utilizing the knowledge of pre-mentioned literature, the aim of current research is:

- To solve numerically the flow and heat exchange for couple stress nanofluid with entropy generation in existence of applied magnetic field and viscous dissipation.

- Formulated governing model is fabricated introducing long wavelength and creeping Stokesian flow approximation which are then analyzed numerically by utilizing Adams Bashforth method.

- Results are demonstrated to examine the behaviors of flow profiles and entropy generation number for emerging flow parameters with the help of graphs, bar-charts and tables.

\section{Problem development and governing model}

Assume the peristaltic motion of incompressible couple stress $(\mathrm{Cu} /$ water) nanofluids conducted in an endoscope. Along the tube wall, sinusoidal wave is transmitting with a uniform speed $c$. Cylindrical coordinate structure $(R$, $Z$ ) is preferred where $Z$-axis is passing through the central line and $R$ indicates the radial direction as depicted in Fig. 1a, whereas the completer flow chart architecture is represented via Fig. 1b. For $a_{1}$ and $a_{2}$ being radii of internal and external tubes in endoscope, wave forms in fixed frame are written as ${ }^{31,32}$ :

$$
\begin{gathered}
r_{1}=a_{1}, \\
r_{2}=a_{2}+b \sin \frac{2 \pi}{\lambda}(Z-c t) .
\end{gathered}
$$

In which $\lambda$ is the wavelength, $b$ is the amplitude of wave and $t$ represents time of travelling wave. Moreover, $T_{1}$ and $T_{0}$ denote temperature of internal and external cylinders, accordingly.

The constitutive equations governing couple stress tensor $M$ and stress tensor $\tau$ are formulated as ${ }^{7,8,32}$ :

$$
\begin{gathered}
\tau=\left(-P+\lambda_{1} \nabla \cdot \bar{q}\right) I+\mu\left[\nabla \bar{q}+(\nabla \bar{q})^{T}\right]+\frac{1}{2} I \times(\nabla \cdot M+\rho C), \\
M=m I+2 \eta \nabla(\nabla \times \bar{q})+2 \eta^{\prime}(\nabla(\nabla \times \bar{q}))^{T} .
\end{gathered}
$$

In which, $m$ denotes the $\frac{1}{3}$ trace of $M, \mu$ and $\lambda_{1}$ express coefficients of viscosity, $C$ indicates vector of body couple while $\eta$ and $\eta^{\prime}$ stand for coefficients of couple stress viscosity. The inequalities constraints for these material constants are given as:

$$
\mu \geq 0,3 \lambda_{1}+2 \mu \geq 0, \eta \geq 0,\left|\eta^{\prime}\right| \leq \eta .
$$

In view of the above relations (3) and (4), the simplified equations in fixed frame of reference are ${ }^{36}$ :

$$
\begin{gathered}
\frac{\partial U}{\partial R}+\frac{U}{R}+\frac{\partial W}{\partial Z}=0 \\
\rho_{n f}\left(\frac{\partial U}{\partial t}+U \frac{\partial U}{\partial R}+W \frac{\partial U}{\partial Z}\right)=-\frac{\partial P}{\partial R}+\mu_{n f}\left(\frac{\partial^{2} U}{\partial R^{2}}+\frac{1}{R} \frac{\partial U}{\partial R}-\frac{U}{R^{2}}+\frac{\partial^{2} U}{\partial Z^{2}}\right) \\
-\eta\left(\frac{\partial^{4} U}{\partial R^{4}}+2 \frac{\partial^{4} U}{\partial R^{2} \partial Z^{2}}+\frac{\partial^{4} U}{\partial Z^{4}}+\frac{2}{R} \frac{\partial^{3} U}{\partial R^{3}}+\frac{2}{R} \frac{\partial^{3} U}{\partial R \partial Z^{2}}-\frac{3}{R^{2}} \frac{\partial^{2} U}{\partial R^{2}}-\frac{2}{R^{2}} \frac{\partial^{2} U}{\partial Z^{2}}+\frac{3}{R^{3}} \frac{\partial U}{\partial R}-\frac{3}{R^{4}}\right), \\
\rho_{n f\left(\frac{\partial W}{\partial t}+U \frac{\partial W}{\partial R}+W \frac{\partial W}{\partial Z}\right)=}-\frac{\partial P}{\partial Z}+\mu_{n f}\left(\frac{\partial^{2} W}{\partial R^{2}}+\frac{1}{R} \frac{\partial W}{\partial R}+\frac{\partial^{2} W}{\partial Z^{2}}\right) \\
-\eta\left(\frac{\partial^{4} W}{\partial R^{4}}+\frac{\partial^{4} W}{\partial R^{2} \partial Z^{2}}+\frac{\partial^{4} W}{\partial Z^{4}}+\frac{2}{R} \frac{\partial^{3} W}{\partial R^{3}}+\frac{2}{R} \frac{\partial^{3} W}{\partial R \partial Z^{2}}-\frac{1}{R^{2}} \frac{\partial^{2} U}{\partial R^{2}}+\frac{1}{R^{3}} \frac{\partial W}{\partial R}\right) \\
+g\left(\rho \beta_{T}\right)_{n f}\left(\mathrm{~T}-\mathrm{T}_{0}\right)-\sigma_{n f} B_{0}^{2} W,
\end{gathered}
$$




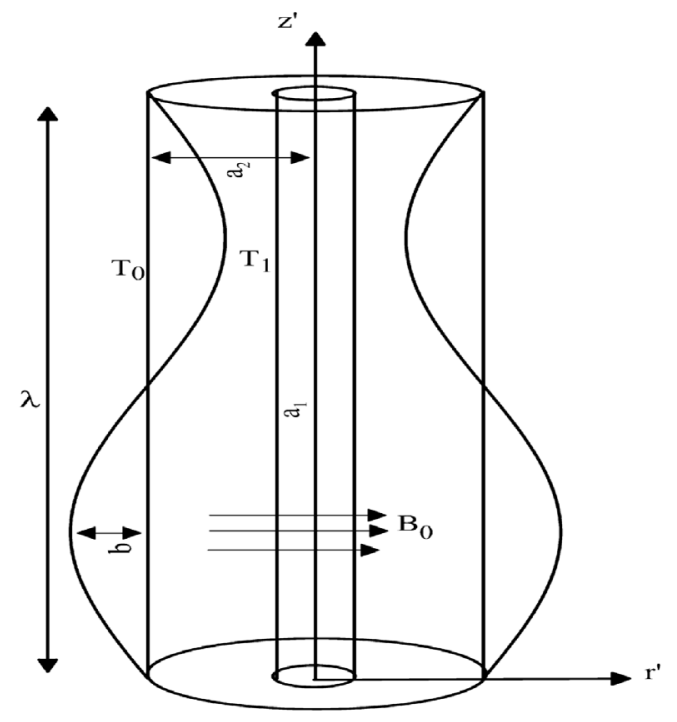

a

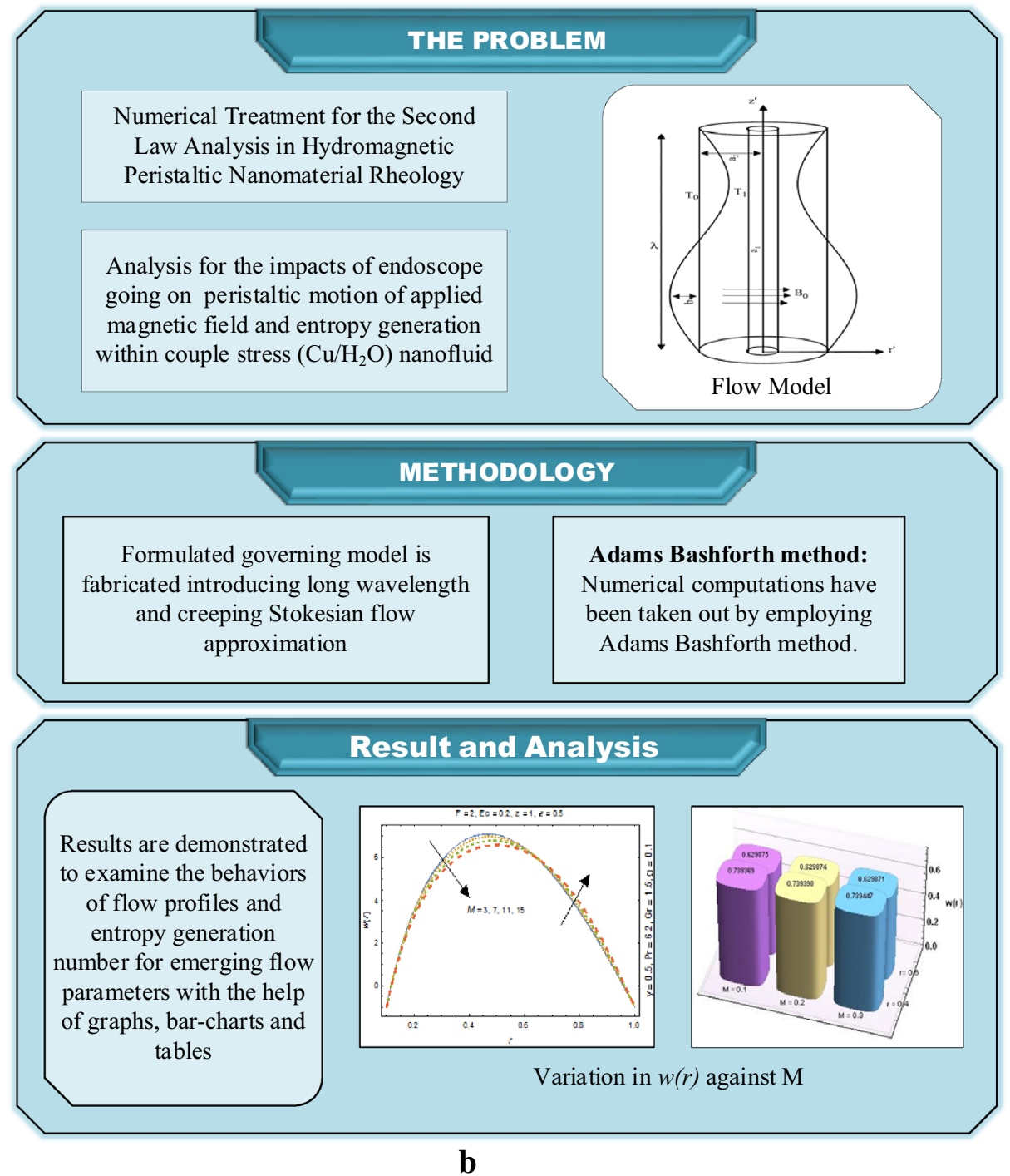

Figure 1. (a) Geometrical representation of flow problem. (b) Process flow architecture. 


$$
\left(\rho c_{p}\right)_{n f}\left(\frac{\partial T}{\partial t}+U \frac{\partial T}{\partial R}+W \frac{\partial T}{\partial Z}\right)=k_{n f}^{*}\left(\frac{\partial^{2} T}{\partial R^{2}}+\frac{1}{R} \frac{\partial T}{\partial R}+\frac{\partial^{2} T}{\partial Z^{2}}\right)+Q_{0}+\Phi
$$

In aforementioned model, $P$ and $T$ represent temperature and pressure of fluid whereas $U$ and $W$ express the $R$ and $Z$ components of velocity, respectively. Further, $g$ expresses gravitational acceleration, $B_{0}$ indicates intensity of external applied magnetic field, $\Phi$ denotes dissipation function and $Q_{0}$ is heat generation parameter.

In the laboratory frame $(R, Z)$, flow is unsteady. In order to obtain a steady flow, transformations of quantities from the laboratory structure $(R, Z)$ to the wave structure $(\mathrm{r}, \mathrm{z}) \operatorname{are}^{7,32}$ :

$$
r=R, \mathrm{z}=\mathrm{Z}-\mathrm{c} t, \mathrm{u}=\mathrm{U}, \mathrm{w}=\mathrm{W}-\mathrm{c}, \mathrm{p}=\mathrm{P} .
$$

where, $u$ and $v$ denote the velocity components in the wave frame $(r, z)$. Equations (5)-(8) yields:

$$
\begin{gathered}
\frac{\partial u}{\partial r}+\frac{u}{r}+\frac{\partial w}{\partial z}=0 \\
A^{*}\left(u \frac{\partial u}{\partial r}+(w+c) \frac{\partial u}{\partial z}\right)=-\frac{1}{\rho_{f}} \frac{\partial p}{\partial r}+\frac{\mu_{f}}{\rho_{f}} \frac{1}{A_{1}}\left(\frac{\partial^{2} u}{\partial r^{2}}+\frac{1}{r} \frac{\partial u}{\partial r}-\frac{u}{r^{2}}+\frac{\partial^{2} u}{\partial z^{2}}\right) \\
-\eta\left(\frac{\partial^{4} u}{\partial r^{4}}+2 \frac{\partial^{4} u}{\partial r^{2} \partial z^{2}}+\frac{\partial^{4} u}{\partial z^{4}}+\frac{2}{r} \frac{\partial^{3} u}{\partial r^{3}}+\frac{2}{r} \frac{\partial^{3} u}{\partial r \partial z^{2}}-\frac{3}{r^{2}} \frac{\partial^{2} u}{\partial r^{2}}-\frac{2}{r^{2}} \frac{\partial^{2} u}{\partial z^{2}}+\frac{3}{r^{3}} \frac{\partial u}{\partial r}-\frac{3}{r^{4}} u\right), \\
A^{*}\left(u \frac{\partial w}{\partial r}+(w+c) \frac{\partial w}{\partial z}\right)=-\frac{1}{\rho_{f}} \frac{\partial p}{\partial z}+\frac{\mu_{f}}{\rho_{f}} \frac{1}{A_{1}}\left(\frac{\partial^{2} w}{\partial r^{2}}+\frac{1}{r} \frac{\partial w}{\partial r}+\frac{\partial^{2} w}{\partial z^{2}}\right) \\
-\eta\left(\frac{\partial^{4} w}{\partial r^{4}}+\frac{\partial^{4} w}{\partial r^{2} \partial z^{2}}+\frac{\partial^{4} w}{\partial z^{4}}+\frac{2}{r} \frac{\partial^{3} w}{\partial r^{3}}+\frac{2}{r} \frac{\partial^{3} w}{\partial r \partial z^{2}}-\frac{1}{r^{2}} \frac{\partial^{2} u}{\partial r^{2}}+\frac{1}{r^{3}} \frac{\partial w}{\partial r}\right) \\
+g\left(\rho \beta_{T}\right)_{f} A_{2}\left(T-T_{0}\right)+\sigma_{f} A_{4} B_{0}^{2}(w+c), \\
A_{5}\left(u \frac{\partial T}{\partial r}+(w+c) \frac{\partial T}{\partial z}\right)=\frac{k_{f}}{\left(\rho c_{p}\right)_{f}} A_{3}\left(\frac{\partial^{2} T}{\partial r^{2}}+\frac{1}{r} \frac{\partial T}{\partial r}+\frac{\partial^{2} T}{\partial z^{2}}\right)+\frac{Q_{0}}{\left(\rho c_{p}\right)_{f}}+\Phi .
\end{gathered}
$$

where, constants involved in the above model are:

$$
\begin{gathered}
A^{*}=(1-\phi)+\phi \frac{\rho_{s}}{\rho_{f}}, A_{1}=(1-\phi)^{2.5}, A_{2}=(1-\phi)+\phi \frac{\left(\rho \beta_{T}\right)_{s}}{\left(\rho \beta_{T}\right)_{f}}, \\
A_{3}=\frac{k_{s}+2 k_{f}-2 \phi\left(k_{f}-k_{s}\right)}{k_{s}+2 k_{f}+2 \phi\left(k_{f}-k_{s}\right)}, A_{4}=(1-\phi)+\phi \frac{\sigma_{s}}{\sigma_{f}}, A_{5}=(1-\phi)+\phi \frac{\left(\rho c_{p}\right)_{s}}{\left(\rho c_{p}\right)_{f}} .
\end{gathered}
$$

where $\phi$ is volume fraction of nanoparticles whereas $\mu_{n f}, \rho_{n f},\left(\beta_{T}\right)_{n f}, \sigma_{n f}$ and $k_{n f}^{*}$ symbolizes the dynamic viscosity, density, thermal expansion coefficient, electric conductivity and thermal conductivity of nanofluid. Moreover, $\mu_{n f}, \rho_{s},\left(\beta_{T}\right)_{s}, \sigma_{s}$ and $k_{s}$ are dynamic viscosity, density, thermal expansion coefficient, electric conductivity and thermal conductivity of nanoparticles while $\mu_{f}, \rho_{f}, k_{f}, \sigma_{f}$ and $\left(\beta_{T}\right)_{f}$ represents base liquid viscosity, density, thermal conductivity, electric conductivity and thermal expansion coefficient, respectively. Now, introducing dimensionless variables as ${ }^{35}$ :

$$
\begin{gathered}
\bar{r}=\frac{r}{a_{2}}, \overline{r_{1}}=\frac{r_{1}}{a_{2}}=c<1, \overline{r_{2}}=\frac{r_{2}}{a_{2}}=1+\phi \sin (2 \pi z), \bar{u}=\frac{\lambda}{a_{2} c} u, \bar{z}=\frac{z}{\lambda}, \phi=\frac{b}{a_{2}}<1, \\
\bar{w}=\frac{w}{c}, \delta=\frac{a_{2}}{\lambda}, \bar{t}=\frac{c t}{\lambda}, \operatorname{Re}=\frac{\rho_{f} c a_{2}}{\mu_{f}}, v=\frac{\mu_{f}}{\rho_{f}}, G r=\frac{\rho_{f} g\left(\beta_{T}\right)_{f} a_{2}^{2}\left(T_{1}-T_{0}\right)}{\mu_{f} c}, \\
\bar{p}=\frac{a_{2}^{2}}{\lambda \mu_{f} c} p, \beta=\frac{Q_{0} a_{2}^{2}}{k_{f}\left(T_{1}-T_{0}\right)}, \theta=\frac{\left(T-T_{0}\right)}{\left(T_{1}-T_{0}\right)}, \mathrm{y}=\sqrt{\frac{\eta}{\mu_{f} a_{2}^{2}}}, \operatorname{Pr}=\frac{\mu_{f}\left(c_{p}\right)_{f}}{k_{f}}, \\
\bar{\psi}=\frac{\psi}{a_{2} c}, \bar{u}=-\frac{\delta}{\bar{r}} \frac{\partial \psi}{\partial \bar{z}}, \bar{w}=\frac{1}{\bar{r}} \frac{\partial \bar{\psi}}{\partial \bar{r}}, \mathrm{Ec}=\frac{c^{2}}{\left(c_{p}\right)_{f}\left(T_{1}-T_{0}\right)}, \mathrm{M}^{2}=\frac{\sigma_{f} B_{0}^{2} a_{2}^{2}}{\mu_{f}} .
\end{gathered}
$$

With the help of long wavelength and creeping flow approximations Eq. (11) is identically fulfilled while Eqs. (12)-(14) reduces to the following expressions: 


$$
\begin{gathered}
\frac{\partial p}{\partial r}=0 \\
y^{2}\left(\frac{\partial^{2}}{\partial r^{2}}+\frac{1}{r} \frac{\partial}{\partial r}\right)^{2}\left(\frac{1}{r} \frac{\partial \psi}{\partial r}\right)-\frac{1}{A_{1}}\left(\frac{\partial^{2}}{\partial r^{2}}+\frac{1}{r} \frac{\partial}{\partial r}\right)\left(\frac{1}{r} \frac{\partial \psi}{\partial r}\right)=-\frac{1}{A_{1}} \frac{\partial p}{\partial z}+A_{2} G r \theta-A_{4} M^{2}\left(\frac{1}{r} \frac{\partial \psi}{\partial r}+1\right),
\end{gathered}
$$

By differentiating Eq. (18) with respect to $r$, we get the following expression:

$$
\begin{gathered}
y^{2} \frac{\partial}{\partial r}\left(\frac{\partial^{2}}{\partial r^{2}}+\frac{1}{r} \frac{\partial}{\partial r}\right)^{2}\left(\frac{1}{r} \frac{\partial \psi}{\partial r}\right)-\frac{1}{A_{1}} \frac{\partial}{\partial r}\left(\frac{\partial^{2}}{\partial r^{2}}+\frac{1}{r} \frac{\partial}{\partial r}\right)\left(\frac{1}{r} \frac{\partial \psi}{\partial r}\right)-A_{2} G r \frac{\partial \theta}{\partial r} \\
+A_{4} M^{2} \frac{\partial}{\partial r}\left(\frac{1}{r} \frac{\partial \psi}{\partial r}+1\right)=0 \\
A_{3}\left(\frac{\partial^{2} \theta}{\partial r^{2}}+\frac{1}{r} \frac{\partial \theta}{\partial r}\right)+\beta+A_{1} E c \operatorname{Pr}\left(\frac{-1}{r^{2}} \frac{\partial \psi}{\partial r}+\frac{1}{r} \frac{\partial^{2} \psi}{\partial r^{2}}\right)^{2}=0 .
\end{gathered}
$$

Bar notation is ignored. Here, $R e, E c, P r$ and $G r$ indicates Reynolds number, Eckert number, Prandtl number and thermal Grashof number whereas $\delta$ expresses wave number and $\theta$ is the dimensionless temperature. Moreover, $\psi$ is stream function for which components of velocity are derived as:

$$
u=\frac{1}{r} \frac{\partial \psi}{\partial r}, v=-\frac{\delta}{r} \frac{\partial \psi}{\partial z} .
$$

The dimensionless boundary conditions are:

$$
\begin{aligned}
& \frac{1}{r} \frac{\partial \psi}{\partial r}=-1, \frac{\partial^{2}}{\partial r^{2}}\left(\frac{1}{r} \frac{\partial \psi}{\partial r}\right)=0, \psi=\frac{-F}{2}, \theta=1, \text { at } \mathrm{r}=\mathrm{r}_{1}, \\
& \frac{1}{r} \frac{\partial \psi}{\partial r}=-1, \frac{\partial^{2}}{\partial r^{2}}\left(\frac{1}{r} \frac{\partial \psi}{\partial r}\right)=0, \psi=\frac{F}{2}, \theta=0, \text { at } \mathrm{r}=\mathrm{r}_{2} .
\end{aligned}
$$

\section{Analysis of entropy generation}

Entropy is the measure of molecular disorder. The volumetric entropy generation rate can be expressed as $^{31}$ :

$$
\dot{S}_{G e n}^{\prime \prime \prime}=\frac{\kappa_{n f}}{T_{0}^{2}}\left(\frac{\partial T}{\partial R}\right)^{2}+\frac{\mu_{n f}}{T_{0}}\left(\frac{\partial W}{\partial R}\right)^{2}+\frac{\sigma B_{0}^{2} W^{2}}{T_{0}} .
$$

Moreover, characteristic entropy generation rate obtained by using boundary conditions in Eq. (23) is:

$$
\dot{S}_{0}^{\prime \prime \prime}=\frac{\kappa_{f}}{T_{0}^{2} a_{2}^{2}}\left(T_{1}-T_{0}\right)^{2},
$$

By using Eq. (24) and dimensionless variables in Eq. (23), dimensionless entropy generation rate is:

$$
N_{s}=\frac{\dot{S}_{G e n}^{\prime \prime \prime}}{\dot{S}_{0}^{\prime \prime \prime}}=A_{3}\left(\frac{\partial \theta}{\partial r}\right)^{2}+\varepsilon E c \operatorname{Pr} \frac{1}{A_{1}}\left(\frac{-1}{r^{2}} \frac{\partial \psi}{\partial r}+\frac{1}{r} \frac{\partial^{2} \psi}{\partial r^{2}}\right)^{2}+\varepsilon E c \operatorname{Pr} M^{2}\left(\frac{1}{r} \frac{\partial \psi}{\partial r}\right)^{2} .
$$

where, $\varepsilon=\frac{T_{0}}{\left(T_{1}-T_{0}\right)}$ represents temperature difference parameter.

\section{Discussion of results}

In this section, effects of important parameters on the velocity and temperature with dimensionless entropy generation number are portrayed graphically. Numerical computations have been taken out by employing Adams Bash forth method that is an iterative technique. The Adams Bash forth methods allow us to compute the approximate solution at a given instant from prior instants' solutions explicitly.

Figures 2 and 3 portray the behavior of velocity toward rising magnitude of velocity parameter $y$ and Grashof number $G$ r. It is noticed that for rising values of both parameters, $w(r)$ augments close the endoscope and declines in the vicinity of the tube walls. The buoyancy forces play a leading effect close to the endoscope and hence fluid velocity upgrades as one move close to the endoscopic tube. Moreover, viscous forces are more dominant near the peristaltic tube so flow rate tends to decrease. Figure 4 explored the oscillatory behavior of $w(r)$ for several values of magnetic parameters $M$. Enlargement in velocity of the fluid near the endoscope is due to dominant effects of Lorentz force which slowly reduces near the tube wall because of no slip condition. Variational trend of temperature of the fluid is explored in Figs. 5, 6, and 7. Increment in magnitude of magnetic parameter causes temperature of the entire fluid to decrease as plotted in Fig. 5. Physics behind such behavior is increasing resistive effects of Lorentz force. Figures 6 and 7 reveal that temperature increases for rising values of $\Omega$ and Ec. Higher values of $\Omega$ yield heat generation while increment in $E c$ leads to higher kinetic energy due to which temperature 


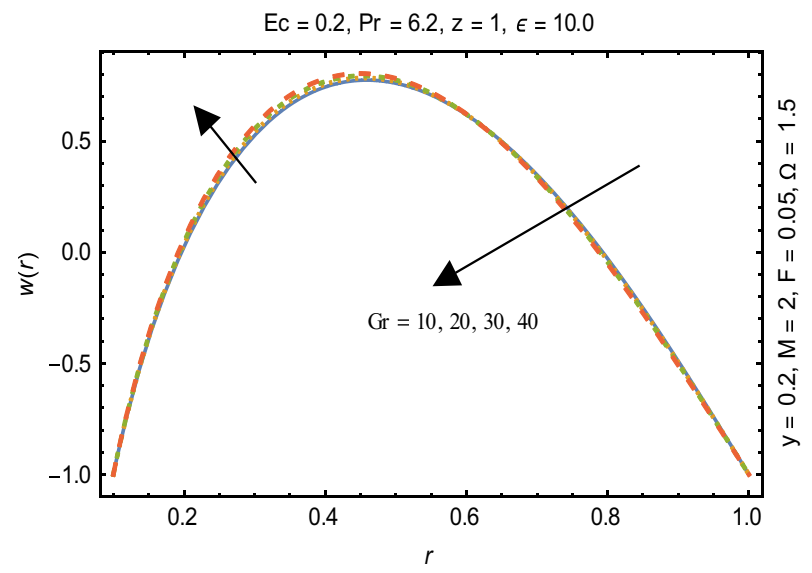

Figure 2. Deviation in $w(r)$ against $\mathrm{G} r$.

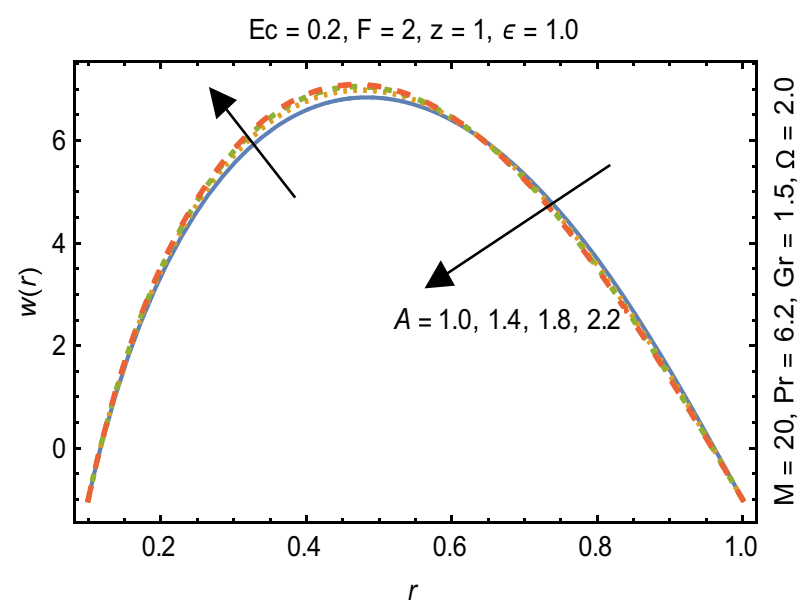

Figure 3. Deviation of $w(r)$ against $\mathrm{y}$.

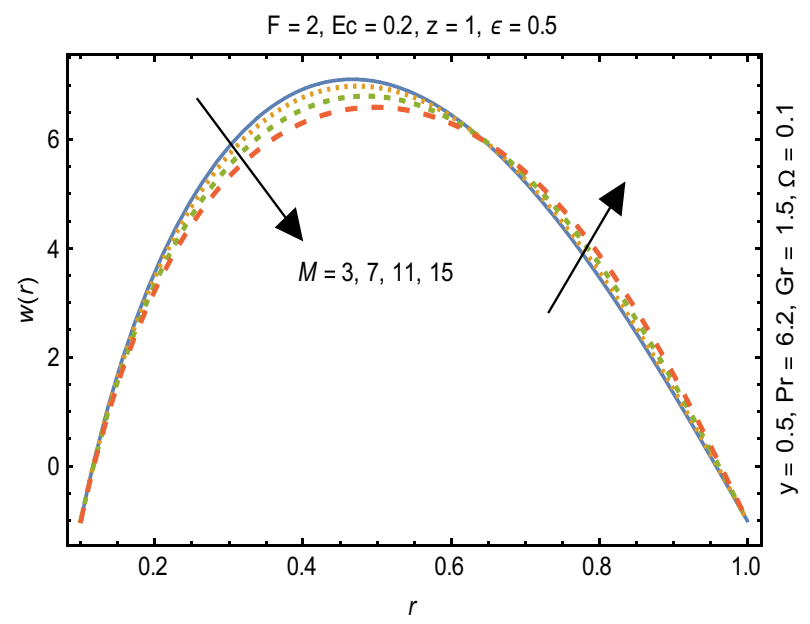

Figure 4. Variation in $w(r)$ against $\mathrm{M}$. 


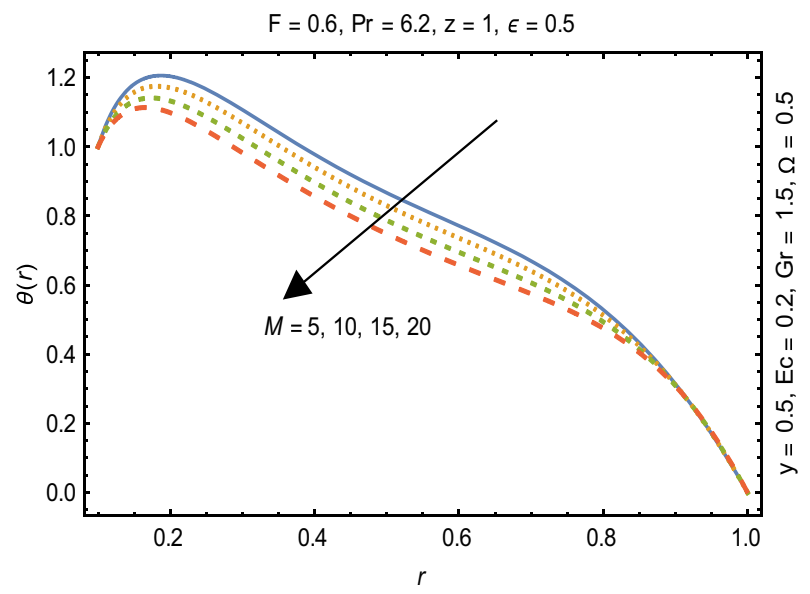

Figure 5. Variation in $\theta(r)$ against $M$.

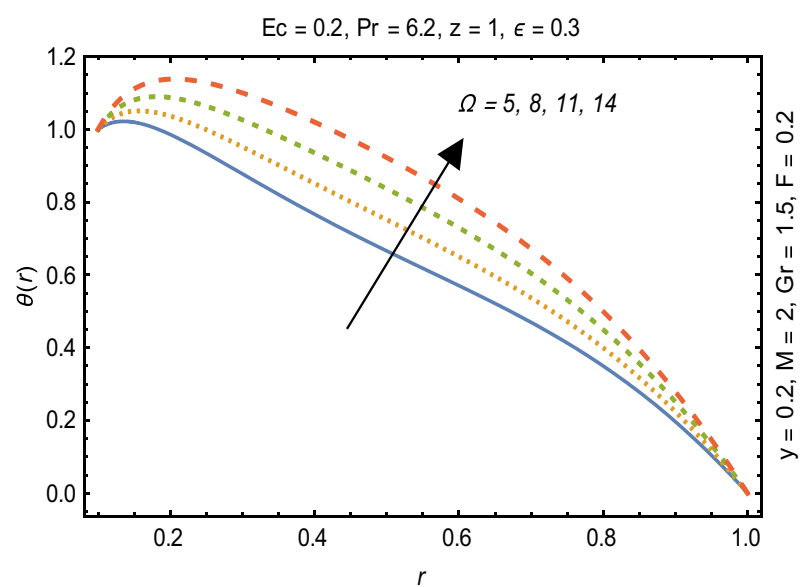

Figure 6. Variation in $\theta(r)$ against $\Omega$.

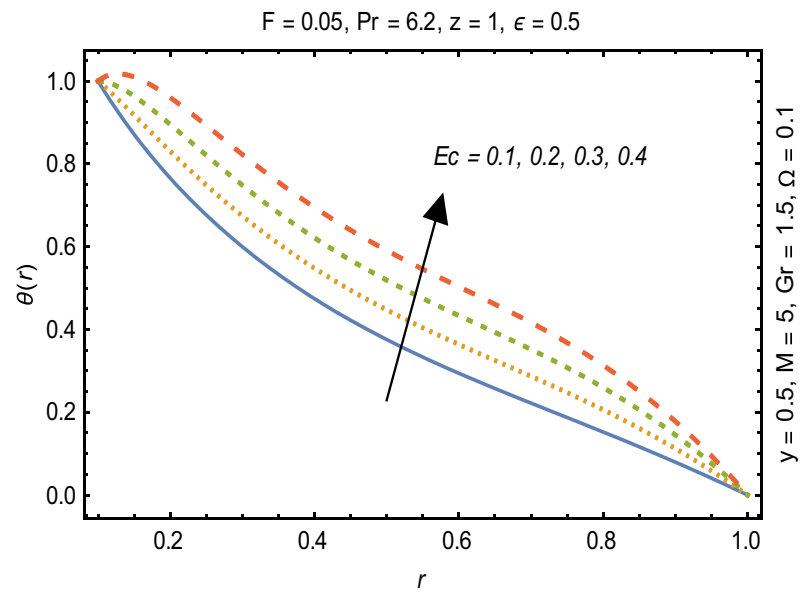

Figure 7. Variation in $\theta(r)$ against Ec. 


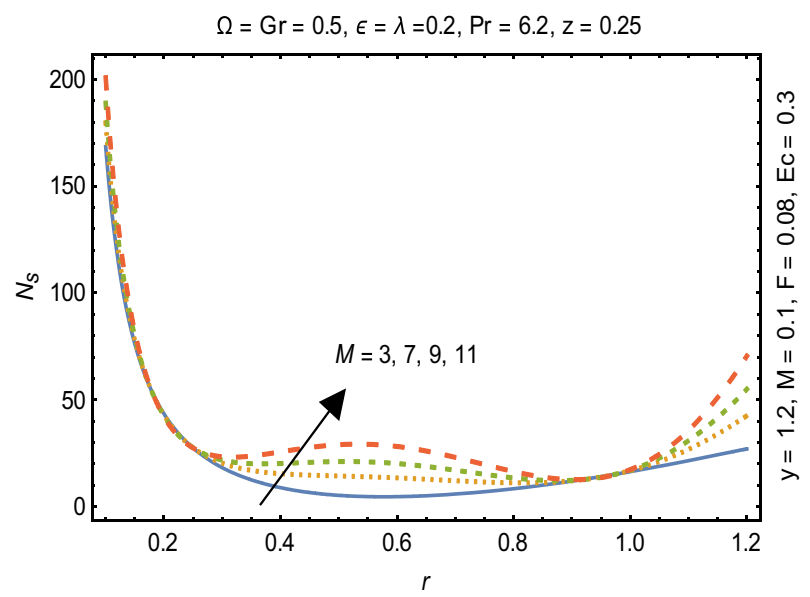

Figure 8. Variation in $N_{s}$ for $\mathrm{M}$.

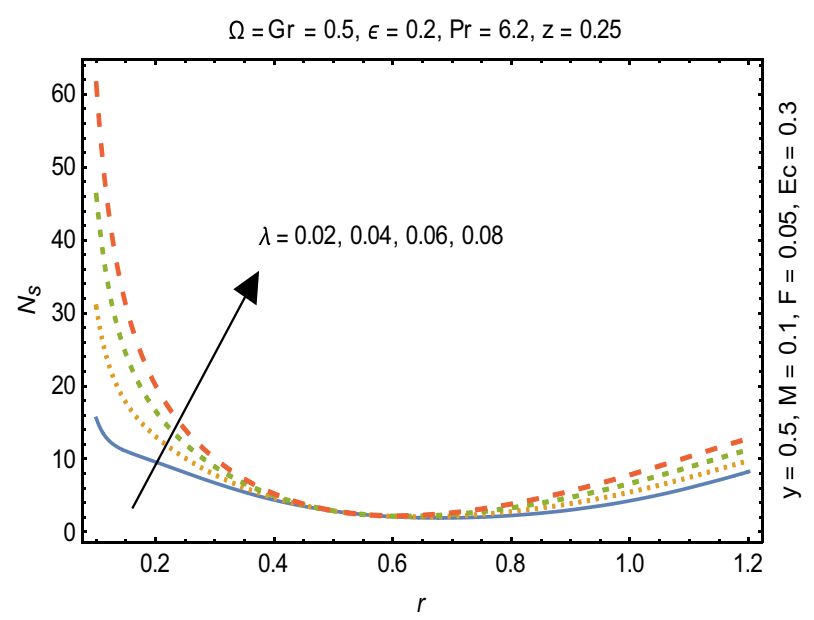

Figure 9. Variation in $N_{s}$ for $\lambda$.

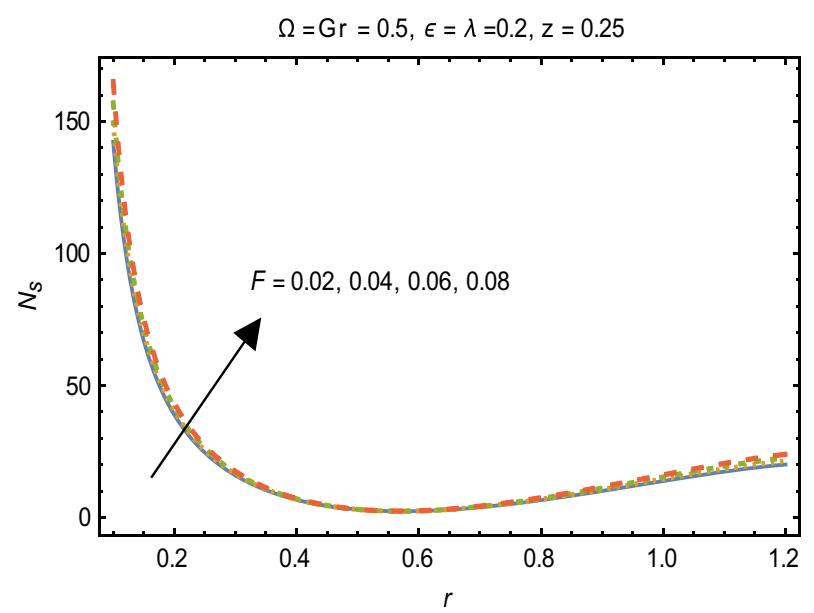

Figure 10. Variation in $N_{s}$ for $F$. 


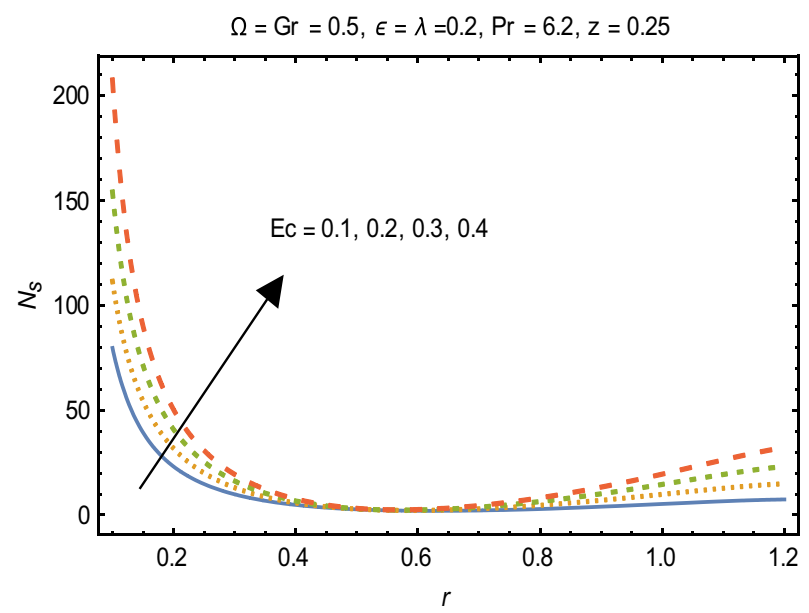

Figure 11. Variation in $N_{s}$ for $E c$.

\begin{tabular}{|l|l|l|}
\hline Properties/constituents & $\mathbf{H}_{2} \mathbf{O}$ & $\mathbf{C u}$ \\
\hline Density, $\rho\left(\mathrm{Kg} / \mathrm{m}^{3}\right)$ & 997 & 8933 \\
\hline Specific heat, $\mathrm{C}_{\mathrm{p}}(\mathrm{J} / \mathrm{kg} \mathrm{K})$ & 4179 & 385 \\
\hline Thermal conductivity, $\kappa(\mathrm{W} / \mathrm{m} \mathrm{K})$ & 0.613 & 401 \\
\hline Thermal expansion coefficient, $\beta\left(10^{-6} \mathrm{~m} /(\mathrm{mK})\right)$ & 210 & 16.65 \\
\hline Electrical conductivity, $\sigma(\mathrm{S} / \mathrm{m})$ & 0.05 & $5.96 \times 10^{7}$ \\
\hline
\end{tabular}

Table 1. Empirical values of thermophysical features for base fluid (water) and nanoparticles (copper) ${ }^{23}$.

\begin{tabular}{|l|l|}
\hline Properties & Nanofluid \\
\hline Density & $\rho_{n f}=\rho_{f}\left[(1-\phi)+\phi \frac{\rho_{s}}{\rho_{f}}\right]$ \\
\hline Heat capacity & $\left(\rho c_{p}\right)_{n f}=\left(\rho c_{p}\right)_{f}\left[(1-\phi)+\phi \frac{\left(\rho c_{p}\right)_{s}}{\left(\rho c_{p}\right)_{f}}\right]$ \\
\hline Viscosity & $\mu_{n f}=\frac{\mu_{f}}{(1-\phi)^{2.5}}$ \\
\hline Thermal conductivity & $\frac{k_{n f}^{*}}{k_{f}}=\frac{k_{s}+2 k_{f}-2 \phi\left(k_{f}-k_{s}\right)}{k_{s}+2 k_{f}+2 \phi\left(k_{f}-k_{s}\right)}$ \\
\hline Thermal expansion coefficient & $\left(\rho \beta_{T}\right)_{n f}=\left(\rho \beta_{T}\right)_{f}\left[(1-\phi)+\phi \frac{\left(\rho \beta_{T}\right)_{s}}{\left(\rho \beta_{T}\right)_{f}}\right]$ \\
\hline Electric conductivity & $\sigma_{n f}=\sigma_{f}\left[(1-\phi)+\phi \frac{\sigma_{s}}{\sigma_{f}}\right]$ \\
\hline
\end{tabular}

Table 2. Expressions for thermophysical features for nanoparticles (copper).

of the fluid rises. Behavior of entropy generation number $N s$ towards physical parameters is illustrated graphically and plotted in Figs. 8, 9, 10, and 11. Magnitude of entropy generation number towards $M$ is increasing near the walls and in the central region including points of intersection at which entropy remains constant. This trend is due to enhancing frictional effects of Lorentz force (Fig. 8).

Figure 9 explicates that higher magnitude of temperature difference parameter has an impact of increasing irreversibility. Physically, it is due to high temperature gradient close to the boundaries. A similar behavior of Ns for $F$ and $E c$ is observed from Figs. 10 and 11 that are caused by no slip wall conditions and thus large velocity gradients.

Further, numerical values for thermophysical properties of nanofluid along with empirical formulas are presented in Tables 1 and 2. Tabulated observations of velocity and temperature against rising values of nondimensional parameters are displayed in Tables 3 and 4 . Moreover, bar charts are drawn for a detailed view. Both of the tabulated as well as bar chart view reveal that with the variations in $G r$ and $M$, magnitude of $w(r)$ increases at $r=0.4$ (near endoscope) and decreases at $r=0.6$ (near tube). These behaviors clearly satisfied the graphical results. Except this, $\theta(r)$ rises for higher magnitudes of Eckert number and magnetic parameter.

Figures 12 and 13 are prepared to present the bar-charts showing the influence of $w(r)$ against $G r$ and $M$ respectively. It is observed that for radial value $r=0.4$ results are significant as compared to $r=0.6$. Moreover Figs. 14 and 15 elucidate the effects of temperature profile $\theta(r)$ vs $E c$ and $M$. We noticed that temperature profile $\theta(r)$ signifies rapidly for larger values of Ec near the walls whereas temperature profile $\theta(r)$ has qualitatively similar behavior for different values of $M$. Figure 16 is constructed for the streamlines of the presented fluid flow 


\begin{tabular}{|l|l|l|l|l|l|l|l|}
\hline \multicolumn{6}{|l}{$\mathbf{C u} / \mathbf{H}_{2} \mathbf{0}$} \\
\hline $\mathbf{M}$ & $\mathbf{F}$ & $\mathbf{E c}$ & $\mathbf{G r}$ & $\mathbf{w}(\mathbf{r}=\mathbf{0 . 2})$ & $\mathbf{w}(\mathbf{r}=\mathbf{0 . 4})$ & $\mathbf{w}(\mathbf{r}=\mathbf{0 . 6})$ & $\mathbf{w}(\mathbf{r}=\mathbf{0 . 8})$ \\
\hline 0.1 & 0.2 & 0.2 & 0.1 & 0.003951 & 0.739369 & 0.629875 & -0.028079 \\
\hline 0.2 & & & & 0.003980 & 0.739398 & 0.629874 & -0.028096 \\
\hline 0.3 & & & & 0.004029 & 0.739447 & 0.629871 & -0.028125 \\
\hline & 0.2 & & & 0.280305 & 1.21785 & 1.07855 & 0.239451 \\
\hline & 0.4 & & & 0.648593 & 1.85599 & 1.67672 & 0.596190 \\
\hline & 0.6 & & & 1.01689 & 2.49417 & 2.2749 & 0.952926 \\
\hline & & 0.2 & & 0.003951 & 2.49425 & 2.27477 & -0.028079 \\
\hline & & 0.4 & & 0.003930 & 2.49451 & 2.2748 & -0.028063 \\
\hline & & 0.6 & & 0.003909 & 2.49477 & 2.27482 & -0.028047 \\
\hline & & & 0.1 & 0.002273 & 0.738773 & 0.629913 & -0.021832 \\
\hline & & & 0.2 & 0.003137 & 0.738816 & 0.62991 & -0.025778 \\
\hline & & & 0.3 & 0.004566 & 0.738858 & 0.629908 & -0.029528 \\
\hline
\end{tabular}

Table 3. Results for velocity profile against nondimensional parameters.

\begin{tabular}{|l|l|l|l|l|l|l|}
\hline $\mathbf{M}$ & $\mathbf{F}$ & $\mathbf{E c}$ & $\mathbf{G r}$ & $\boldsymbol{\theta}(\mathbf{r}=\mathbf{0 . 2})$ & $\boldsymbol{\theta}(\mathbf{r}=\mathbf{0 . 4})$ & $\boldsymbol{\theta}(\mathbf{r}=\mathbf{0 . 6})$ \\
\hline 0.1 & 0.2 & 0.2 & 0.1 & 1.06589 & 0.544824 & 0.361133 \\
\hline 0.2 & & & & 1.06590 & 0.544827 & 0.361136 \\
\hline 0.3 & & & & 1.06591 & 0.544833 & 0.361142 \\
\hline & 0.2 & & & 0.906249 & 1.27564 & 0.446693 \\
\hline & 0.4 & & & 1.04157 & 1.8217 & 0.592958 \\
\hline & 0.6 & & & 1.21094 & 2.50981 & 0.776021 \\
\hline & & 0.2 & & 1.06589 & 0.544833 & 0.361142 \\
\hline & & 0.4 & & 1.05017 & 0.688935 & 0.497805 \\
\hline & & 0.6 & & 1.03445 & 0.833058 & 0.634488 \\
\hline & & & 0.1 & 1.06081 & 0.544757 & 0.361070 \\
\hline & & & 0.2 & 1.06407 & 0.544762 & 0.361075 \\
\hline & & & 0.3 & 1.06699 & 0.544767 & 0.361079 \\
\hline
\end{tabular}

Table 4. Results for temperature profile against nondimensional parameters.

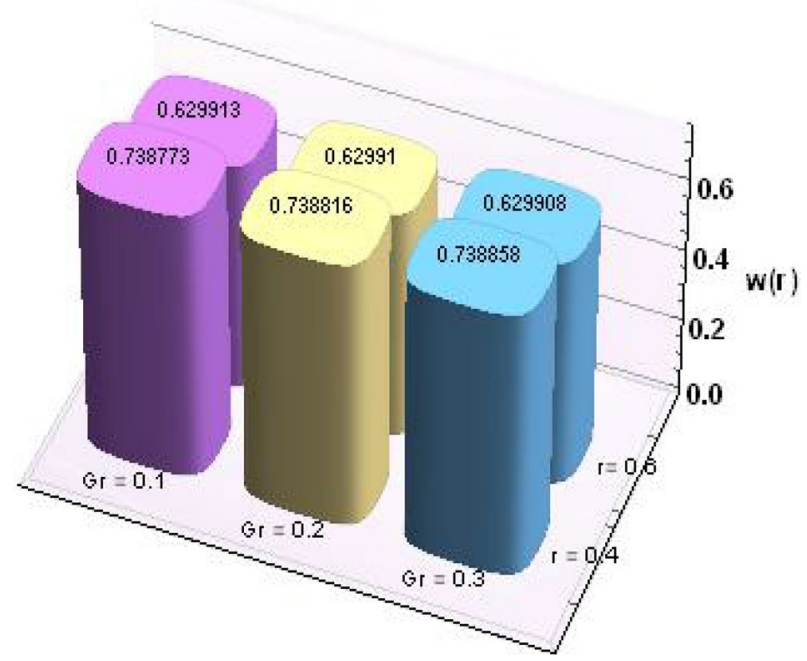

Figure 12. Variation in $w(r)$ against $G r$. 


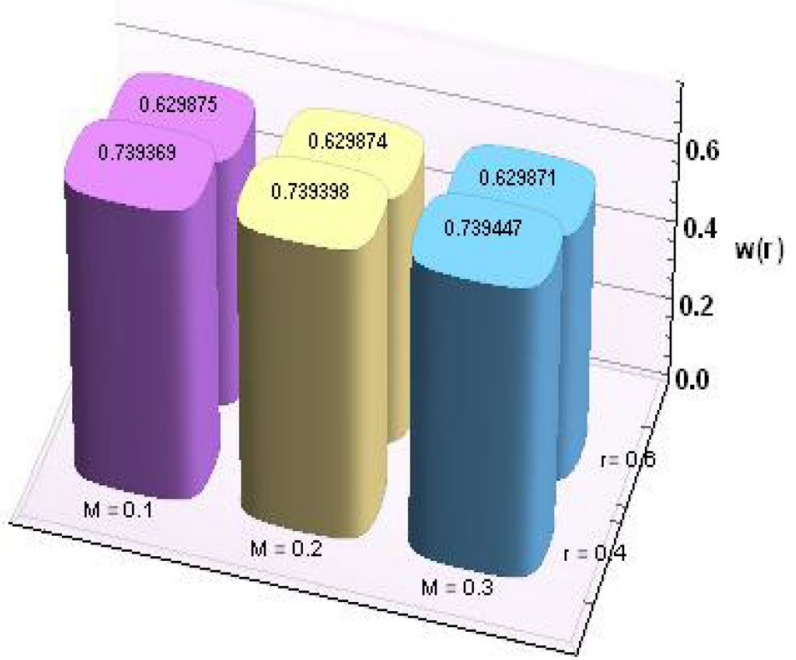

Figure 13. Variation in $w(r)$ against $M$.

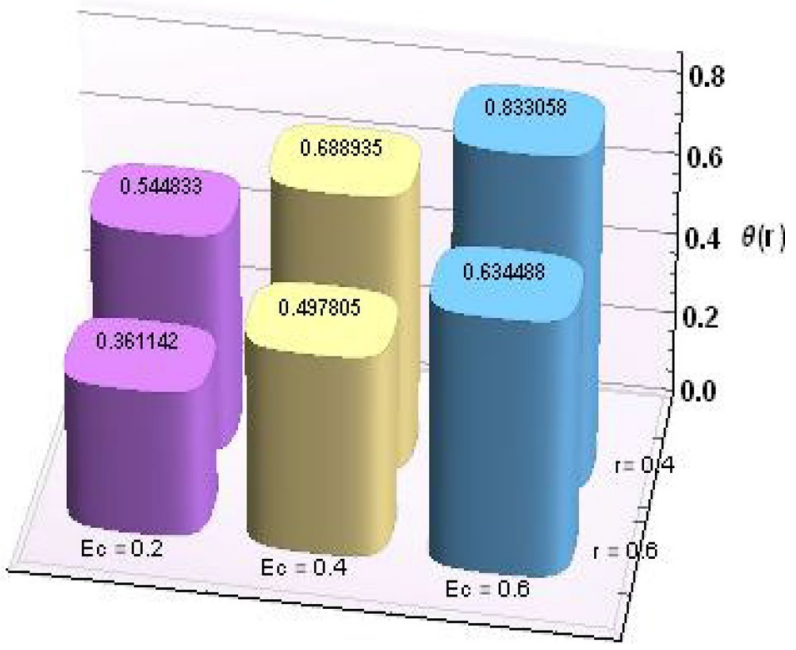

Figure 14. Variation in $\theta(r)$ against $E c$.

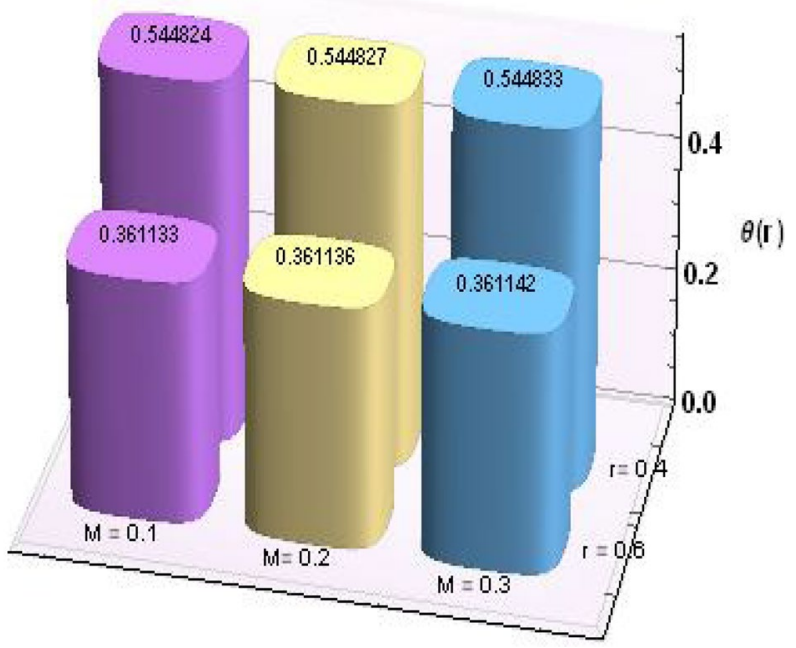

Figure 15. Variation in $\theta(r)$ against $M$. 


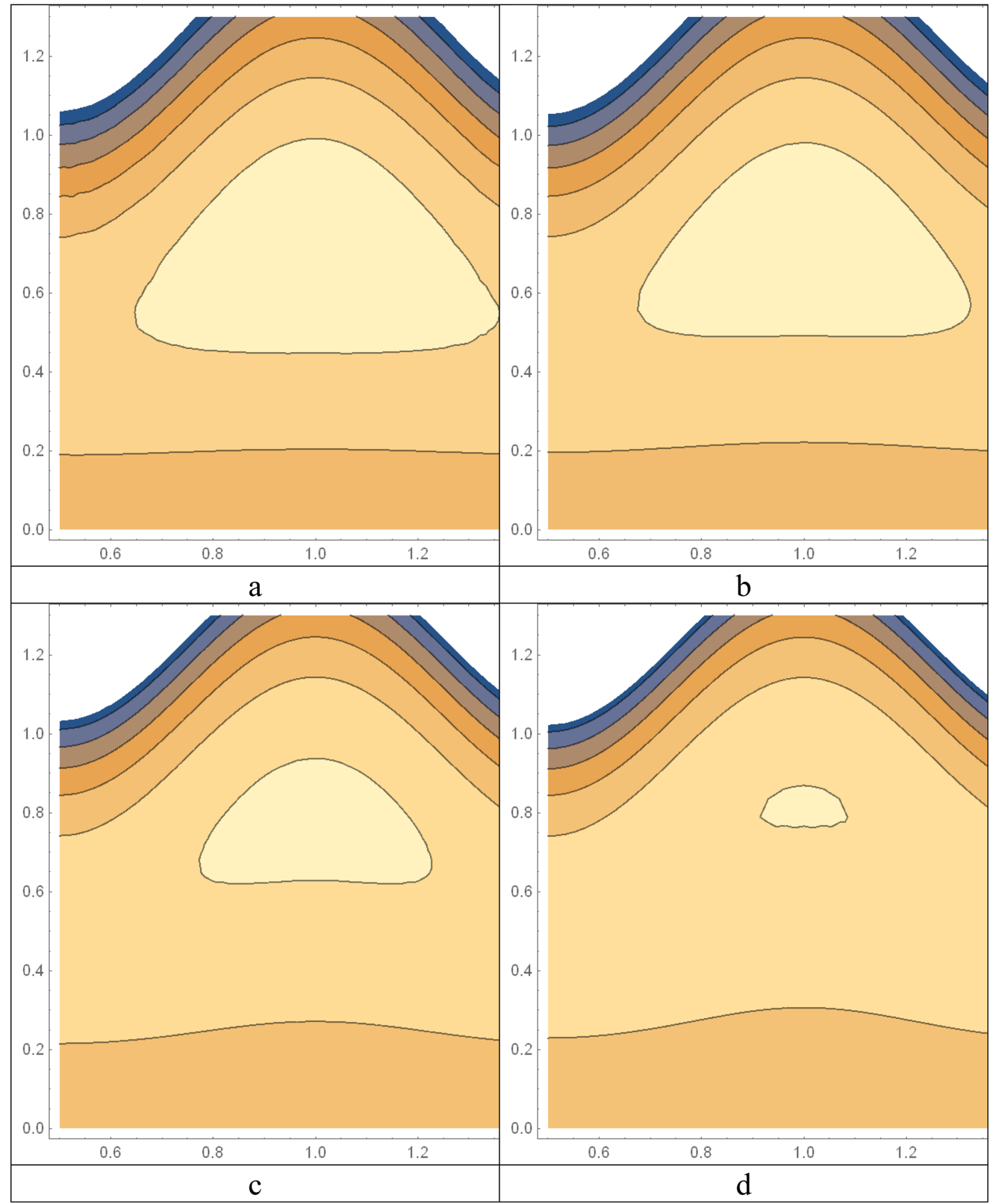

Figure 16. Streamlines for different $M,(\mathbf{a}) M=0.01$, (b) $M=1.0$, (c) $M=2.0$, (d) $M=3.0$.

corresponding to various parameters of interests associated the fluid flow system. The flow pattern is shown by the streamlines presented via Fig. 16. 


\section{Concluding remarks}

In the present research article, analysis for the impacts of endoscope going on peristaltic flow of couple stress nanofluid in the existence of magnetic field and viscous dissipation is carried out. Major conclusions drawn from present investigation are:

- Velocity profile increases close to the endoscope and decreases close to the peristaltic vertical tube with increment of $G r$ and $y$ while an opposite behavior is noticed for $M$.

- Temperature increases against higher values of $\Omega$ and $E c$ but an opposite behavior is depicted for $M$.

- Entropy is directly affected by buoyancy and viscous forces which are dominant near the endoscope and tube walls.

- The consequences of Newtonian fluid model can be obtained by taking the couple stress parameter $y=0$ within the current model.

\section{Future work}

In the future research, the soft computing intelligent techniques can be implemented as an efficient/accurate stochastic numerical solver implemented in nonlinear computational fluid mechanics models ${ }^{33-36}$, singular and multi-singular differential systems $\mathrm{s}^{37-39}$ and mathematical models representing the problems of epidemiology $\mathrm{y}^{40-42}$.

Received: 12 July 2021; Accepted: 28 December 2021

Published online: 28 January 2022

\section{References}

1. Devakar, M. \& Iyengar, T. K. V. Run up flow of an incompressible couple stress fluid between parallel plates. Non Linear Anal. Model. Control 15, 29-37 (2010).

2. Devakar, M., Sreenivasu, D. \& Shankar, B. Analytical solutions of couple stress fluid flows with slip boundary conditions. Alexand. Eng. J. 53, 723-730 (2014).

3. Devakar, M., Sreenivasu, D. \& Shankar, B. Analytical solutions of some fully developed flows of couple stress fluid between concentric cylinders with slip boundary conditions. Int. J. Eng. Math. 53, 396-785 (2014).

4. Srivastava, L. M. Flow of couple stress fluid through stenotic blood vessels. J. Biomech. 18, 479-485 (1985).

5. Srinivasacharya, D. \& Srikanth, D. Steady streaming effect on the flow of a couple stress fluid through a constricted annulus. Arch. Mech. 64, 137-152 (2012).

6. Hayat, T., Awais, M. \& Ambreen, S. A. Unsteady three dimensional flow of couple stress fluid over a stretching surface with chemical reaction. Nonlinear Anal. Modell. Control 17, 47-59 (2012).

7. Awais, M., Saleem, S. \& Hayat, T. Hydromagnetic couple-stress nanofluid flow over a moving convective wall, OHAM analysis. Acta Astronaut. 129, 271-276 (2016).

8. Tripathi, D., Jhorar, R. \& Beg, O. A. Electro-magneto-hydrodynamic peristaltic pumping of couple stress biofluids through a complex wavy micro-channel. J. Modeling Liquid. 236, 358-367 (2017).

9. Murthy, J. V. R. \& Srinivas, J. First and second law analysis for the MHD flow of two immiscible couple stress fluids between two parallel plates. Heat Transf. Asian Res. 43, 1-20 (2014).

10. Tripathi, D., Yadav, A. \& Beg, O. A. Electro-osmotic flow of couple stress fluids in a microchannel propagated by peristalsis. Eur. Phys. J. Plus. 132, 173-185 (2017).

11. Choi, S. U. S. \& Eastman, J. A. Enhancing thermal conductivity of fluids with nano particles. Prog. ASME Int. Mech. Eng. Congress Exposit. 66, 99-105 (1995).

12. Khan, S. U., Shehzad, S. A., Rauf, A. \& Ali, N. Mixed convection flow of couple stress nanofluid over oscillatory stretching sheet with heat absorption/generation effects. Results Phys. 8, 1223-1231 (2018)

13. Khan, N. A., Sultan, F., Riaz, F. \& Jamil, M. Investigation of combined heat and mass transfer between vertical parallel plates in a two layer flow of couple stress nanofluid. Open Eng. 6, 35-43 (2016).

14. Sithole, H., Mondal, H., Goco, S. \& Sibanda, P. Numerical simulation of couple stress nanofluid flow in magneto-porous medium with thermal radiation and a chemical reaction. Appl. Math. Comput. 339, 820-836 (2018).

15. Tayeb, M., Bouaziz, M. N. \& Hanini, S. Influence of non-linear Boussinesq approximation and convective thermal boundary condition on MHD natural convection flow of a couple stress-nanofluid in a porous medium. Nanohybrids Composit. 26, 45-61 (2019).

16. Messaoudi, N., Bouaziz, M. N. \& Agha, H. A. Double diffusive convection in flow of couple stress nanofluid in a permeable wall of vertical channel in the presence of magnetic field. Nano Hybrids Composit. 26, 30-44 (2019).

17. Engelmann, T. W. Zur physiologie des ureter. Pflugera Arch. Physiol. 2, 243-293 (1869).

18. Hayat, T., Yasmin, H., Ahmad, B. \& Chen, B. Simultaneous effects of convective conditions and nanoparticles on the peristaltic motion. J. Mol. Liq. 193, 74-82 (2014).

19. Vajravelu, K., Radhakrishnamacharya, G. \& Radhakrishnamurty, V. Peristaltic flow and heat transfer in a vertical porous annulus with long wave approximation. Int. J. Non-Linear Mech. 42, 754-759 (2007).

20. Shahzadi, I. \& Nadeem, A. Inclined magnetic field analysis for metallic nanoparticles submerged in blood with convective boundary condition. J. Mol. Liq. 230, 61-73 (2017).

21. Hayat, T., Rafiq, M., Ahmad, B. \& Asghar, S. Entropy generation analysis for peristaltic flow of nanoparticles in a rotating frame. Int. J. Heat Mass Transf. 108, 1775-1786 (2017).

22. Jangili, S., Adesanya, S. O., Ogunseye, H. A. \& Lebelo, R. Couple stress fluid flow with variable properties: A second law analysis. Math. Methods Appl. Sci. 42, 85-98 (2019).

23. Abbas, M. A., Bai, Y., Rashidi, M. M. \& Bhatti, M. M. Analysis of entropy generation in the flow of peristaltic nanofluid in channel with complaint walls. Entropy 18, 18030090 (2016).

24. Abbasi, F. M., Shanakhat, I. \& Shehzad, A. M. Entropy generation analysis for peristalsis of nanofluid with temperature dependent viscosity and Hall effects. J. Magn. Matter. 474, 434-441 (2019).

25. Ali, M. et al. Computational analysis of entropy generation for cross-nanofluid flow. Appl. Nanosci. 48, 1-11 (2019).

26. Awan, S. E., Khan, Z. A., Awais, M., Rehman, S. U. \& Raja, M. A. Z. Numerical treatment for hydro-magnetic unsteady channel flow of nanofluid with heat transfer. Results Phys. 9, 1543-1554 (2018).

27. Nawaz, M., Rana, S. \& Qureshi, I. H. Computational fluid dynamics simulations for dispersion of nanoparticles in a magnetohydrodynamic liquid: A Galerkin finite element method. RSC Adv. 8, 38324-38335 (2018).

28. Abbas, M. A., Bai, Y., Rashidi, M. M. \& Bhatti, M. M. Application of drug delivery in megnetohydrodynamics peristaltic blood flow of nano fluid in a non-uniform channel. J. Mech. Med. Biol. 16, 1650052 (2016). 
29. Rana, P. \& Shukla, N. Entropy generation analysis for non-similar analytical study of nanofluid flow and heat transfer under the influence of aligned magnetic field. Alex. Eng. J. 57, 3299-3310 (2018).

30. Sheikholeslami, M. et al. Simulation of convection heat transfer of magnetic nanoparticles including entropy generation using CVFEM. Int. J. Heat Mass Transf. 136, 146-156 (2019).

31. Akbar, N. S., Raza, M. \& Ellahi, R. Endoscopic effects with entropy generation analysis in peristalsis for the thermal conductivity of copper-water nanofluid. J. Appl. Fluid Mech. 9, 1721-1730 (2016).

32. Rameesh, K. \& Devakar, M. Effect of endoscope on the peristaltic transport of a couple stress fluid with heat transfer: Application to biomedicine. Nonlinear Eng. 8, 619-629 (2019).

33. Ilyas, H. et al. A novel design of Gaussian wavelet neural networks for nonlinear Falkner-Skan systems in fluid dynamics. Chin. J. Phys. 72, 386-402 (2021).

34. Shoaib, M. et al. Neuro-computing networks for entropy generation under the influence of MHD and thermal radiation. Surf. Interfaces 25, 101243 (2021).

35. Aljohani, J. L. et al. Intelligent computing through neural networks for numerical treatment of non-Newtonian wire coating analysis model. Sci. Rep. 11(1), 1-32 (2021).

36. Uddin, I. et al. Design of intelligent computing networks for numerical treatment of thin film flow of Maxwell nanofluid over a stretched and rotating surface. Surf. Interfaces 24, 101107 (2021).

37. Sabir, Z. et al. A novel design of fractional Mayer wavelet neural networks with application to the nonlinear singular fractional Lane-Emden systems. Alex. Eng. J. 60(2), 2641-2659 (2021).

38. Sabir, Z., Raja, M. A. Z., Guirao, J. L. \& Shoaib, M. A neuro-swarming intelligence-based computing for second order singular periodic non-linear boundary value problems. Front. Phys. 8, 224 (2020).

39. Sabir, Z. et al. Design of stochastic numerical solver for the solution of singular three-point second-order boundary value problems. Neural Comput. Appl. 2, 1-17 (2020).

40. Ahmad, I. et al. Novel applications of intelligent computing paradigms for the analysis of nonlinear reactive transport model of the fluid in soft tissues and microvessels. Neural Comput. Appl. 31(12), 9041-9059 (2019).

41. Ahmad, I. et al. Integrated neuro-evolution-based computing solver for dynamics of nonlinear corneal shape model numerically. Neural Comput. Appl. 1, 1-17 (2020).

42. Shoaib, M. et al. A stochastic numerical analysis based on hybrid NAR-RBFs networks nonlinear SITR model for novel COVID-19 dynamics. Comput. Methods Programs Biomed. 1, 105973 (2021).

\section{Acknowledgements}

This study was supported by Taif University Researchers Supporting Project Number (TURSP-2020/117), Taif University, Taif, Saudi Arabia. One of the authors (M.Y.Malik) extends his appreciation to the Deanship of Scientific Research at King Khaled University, Abha, 61413, Saudi Arabia for funding this work through research group program under grant number G. R. P-115/1443.

\section{Author contributions}

Conceptualization: M.A., M.S., M.A.Z.R.; Writing Original Draft: M.A., M.S., M.A.Z.R., S.A., M.Y.M., K.S.N.; Software: M.S., S.A., M.Y.M., K.S.N.; Formal Analysis: M.A., M.A.Z.R., S.A., K.A.I.; Validation: M.Y.M., K.S.N.; Revision, review and Editing: M.A., M.S., M.A.Z.R., S.A., M.Y.M., K.S.N., K.A.I.; Funding Acquisition: K.A.I.

\section{Competing interests}

The authors declare no competing interests.

\section{Additional information}

Correspondence and requests for materials should be addressed to M.A.Z.R. or K.S.N.

Reprints and permissions information is available at www.nature.com/reprints.

Publisher's note Springer Nature remains neutral with regard to jurisdictional claims in published maps and institutional affiliations.

Open Access This article is licensed under a Creative Commons Attribution 4.0 International License, which permits use, sharing, adaptation, distribution and reproduction in any medium or format, as long as you give appropriate credit to the original author(s) and the source, provide a link to the Creative Commons licence, and indicate if changes were made. The images or other third party material in this article are included in the article's Creative Commons licence, unless indicated otherwise in a credit line to the material. If material is not included in the article's Creative Commons licence and your intended use is not permitted by statutory regulation or exceeds the permitted use, you will need to obtain permission directly from the copyright holder. To view a copy of this licence, visit http://creativecommons.org/licenses/by/4.0/.

(C) The Author(s) 2022 